\title{
A similarity transformation for inviscid non-Boussinesq gravity currents
}

\author{
A.-C. Ruo and F. Chen, Taipei, Taiwan \\ Received August 9, 2004 \\ Published online: November 19, 2004 (C) Springer-Verlag 2004
}

\begin{abstract}
Summary. We propose a similarity transformation to investigate the motion of inviscid gravity currents in the similar phase. With this similarity transformation, the shallow water equations can be solved without needing to consider the front condition Eq. (1) downstream of the gravity current, and the parameter $\beta$ of Eq. (1) becomes a part of the similarity solutions. It is shown that the resultant similarity solutions are in good agreement with the experimental results of both the dam-break and the lock-exchange nonBoussinesq currents, which have a density much larger than the ambient fluid.
\end{abstract}

\section{Introduction}

The gravity current is a heavier gravity-driven flow intruding beneath a lighter ambient fluid, a phenomenon widely observed in both natural and industrial situations [1], [2]. To analyze the motion of gravity currents, a common approach is to solve the hyperbolic shallow water equations together with the boundary conditions at both the current source upstream and the current front downstream [3]-[9]. Under some circumstances the gravity current may become supercritical and induce a hydraulic jump in the middle of the current, so that the jump condition at the middle of the current should also be taken into account [10].

In spite of the hyperbolic nature of the shallow water equations, the use of the front condition downstream is a "logical reconciliation" [9] because the vertical accelerations are not small at the current front, where the shallow water equations are invalid while a major part of the resistance force from the ambient fluid (or the ambient resistance) is applied, and which to some extent can be accounted for by the front condition. But for some gravity currents, such as the accidental release of toxic liquid gas due to the rupture of a storage tank or the currents generated by a dam-break accident, both important concerning human safety, the density difference between the current and the ambient fluid (or the non-Boussinesq gravity current) is so large that the ambient resistance (or the inertia resistance for inviscid currents without surface tension) is negligible. Consequently, the consideration of the front condition may have lost its logical reason in physical sense.

Moreover, according to the definition of the front condition

$u_{f}=\sqrt{\beta g_{r} h_{f}}$,

where $u_{f}$ is the front speed of the current, $g_{r}=\left[\left(\rho_{1}-\rho_{2}\right) / \rho_{1}\right] g$ is the reduced gravity, $\rho_{1}$ and $\rho_{2}$ are the densities of fluids I and II (see Fig 1), respectively, and $h_{f}$ is the height of the current front, the parameter $\beta$ shall be given as a prerequisite in the analysis. Accordingly, the solutions 


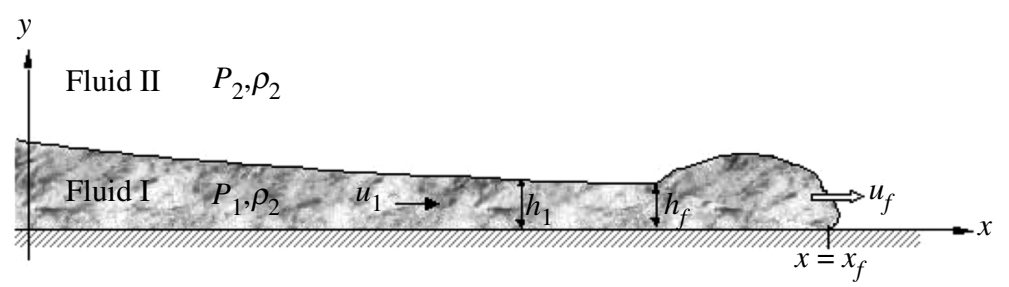

Fig. 1. A schematic description of the two-dimensional inviscid gravity current moving to the right. A fluid of higher density accounts for layer I and the ambient fluid of lower density accounts for layer II. The height of the gravity current is represented by $h_{1}(x, t)$ and the velocity by $u_{1}(x, t)$, and $h_{f}$ and $u_{f}$ at the current front, respectively

are dependent on the value of $\beta$ so that the determination of $\beta$ becomes an important issue of gravity current researches [1], [11], [12]. Even if the front condition Eq. (1) has been widely accepted, the above-mentioned approach is still mathematically "not closed" [5] due to the need of $\beta$ as a prerequisite for analyses.

In the present study, we propose a similarity transformation for inviscid gravity currents, with which the shallow water equations together with the constrained condition at the current source can be solved without needing the front condition Eq. (1), and the value of $\beta$ becomes part of the solution. The resultant similarity solutions turn out to be in good agreement with experimental results for non-Boussinesq currents and can be reduced to those of previous studies employing different similarity transformations and using the front condition Eq. (1) to solve the shallow water equations.

In the following, we derive in Sect. 2 the similarity variables and apply them to solve the shallow water equations without considering the front condition Eq. (1). In Sect. 3, we show that the similarity solutions are in a good agreement with the experimental results of two nonBoussinesq gravity currents: the dam-break and the lock-exchange currents. Finally, conclusions are drawn in Sect. 4.

\section{The similarity solution}

Consider a two-dimensional inviscid gravity current in its self-similar phase (see Fig. 1), where the surface tension is neglected. After applying the shallow water approximations $u=u(x, t)$, $u \gg v, \frac{\partial}{\partial x} \ll \frac{\partial}{\partial y}$ and relevant boundary conditions, the continuity and momentum equations can be reduced to the shallow water equations

$\frac{\partial h_{1}}{\partial t}+\frac{\partial\left(u_{1} h_{1}\right)}{\partial x}=0$

$\frac{\partial u_{1}}{\partial t}+u_{1} \frac{\partial u_{1}}{\partial x}+g_{r} \frac{\partial h_{1}}{\partial x}=0$,

where $u_{1}(x, t)$ is the velocity in the $x$-direction and is uniform over the current depth and $h_{1}(x, t)$ is the height of the gravity current. Equations (2) and (3) are coupled nonlinear partial differential equations, which can be solved by similarity transformation. To obtain the similarity variables, we consider the following forms:

$u_{1}(x, t) \equiv U \tilde{u}(\eta), \quad h_{1}(x, t) \equiv H \tilde{h}(\eta), \quad \eta \equiv m \frac{x}{t^{n}}$. 
The parameters $U, H, m, n$ are determined by the dimensional analysis of Eqs. (2) and (3) after Eq. (4) is applied, yielding

$n=1, \quad m=\left(Q_{\alpha} g_{r}^{2-\alpha}\right)^{\frac{-1}{4-\alpha}}, \quad U=\left(Q_{\alpha} g_{r}^{2-\alpha}\right)^{\frac{1}{4-\alpha}}, \quad H=\left(Q_{\alpha}^{2} g_{r}^{-\alpha}\right)^{\frac{1}{4-\alpha}}$,

where $Q_{\alpha}$ is given in the so-called constrained condition [13]

$\int_{0}^{x_{f}} h_{1}(x, t) d x=Q_{\alpha} t^{\alpha}$,

where $x_{f}$ is the position of the current front. This condition presents the conservation of current volume and $Q_{\alpha} t^{\alpha}$ accounts for the volume of gravity current per unit width. Accordingly, $\alpha=0$ corresponds to the "constant volume" current and $\alpha=1$ to the "constant flux" current [6].

The similarity transformation (4) and (5) is applied to transform the shallow water equations (2) and (3) into ordinary differential equations as follows:

$(\tilde{u}-\eta) \frac{d \tilde{h}}{d \eta}+\tilde{h} \frac{d \tilde{u}}{d \eta}=0$,

$(\tilde{u}-\eta) \frac{d \tilde{u}}{d \eta}+\frac{d \tilde{h}}{d \eta}=0$.

Equation (7) can be rewritten into

$\frac{d \tilde{h}}{d \eta}=-\frac{\tilde{h}(d \tilde{u} / d \eta)}{(\tilde{u}-\eta)}$,

in which $\tilde{u}=\eta$ is a trivial solution leading to $\tilde{h}=0$, so that we shall have $\tilde{u} \neq \eta$. We then substitute Eq. (7) into Eq. (8), yielding

$\left[(\tilde{u}-\eta)^{2}-\tilde{h}\right] \frac{d \tilde{u}}{d \eta}=0$.

If $d \tilde{u} / d \eta=0, \tilde{h}$ will be constant (or a gravity current of uniform height [6]), which is also a trivial solution and is physically not true. Accordingly we shall have $d \tilde{u} / d \eta \neq 0$ and $\tilde{h}=(\tilde{u}-\eta)^{2}$, and then $(\tilde{u}-\eta)^{2}[3(d \tilde{u} / d \eta)-2]=0$ as Eq. (7) is considered. Since $\tilde{u} \neq \eta$, we get

$\tilde{u}=\frac{2}{3} \eta+C, \quad \tilde{h}(\eta)=\left(C-\frac{\eta}{3}\right)^{2}$.

By applying Eq. (4), this can be written as

$u_{1}(x, t)=U \tilde{u}(\eta)=U\left[\frac{2}{3} m \frac{x}{t}+C\right]$,

$h_{1}(x, t)=H \tilde{h}(\eta)=H\left(C-\frac{m}{3 t} x\right)^{2}$,

where $C$ is an integral constant to be determined by the constrained condition Eq. (6). To do this, we substitute Eq. (13) into Eq. (6) and obtain

$x_{f}=3 \frac{t}{m}\left[C+\left(D t^{\alpha-1}-C^{3}\right)^{\frac{1}{3}}\right]$.

where $D=\left(Q_{\alpha}^{1-\alpha} g_{r}^{2 \alpha-2}\right)^{\frac{1}{4-\alpha}}$. By applying Eqs. (12) and (14) on the relation

$u_{f}(t)=\frac{d x_{f}}{d t}$ 
and by assuming that $u_{f}(t)=u_{1}\left(x_{f}, t\right)$, we obtain $\left(D t^{\alpha-1}-C^{3}\right)+D(\alpha-1) t^{\alpha-1}=0$, from which the constant $C$ is obtained as

$C=\left(\alpha t^{\alpha-1} D\right)^{\frac{1}{3}}$.

Note that since $C$ is an integral constant and shall not be a function of time, which can be satisfied only when $\alpha=0$ (yielding $C=0$ ) and $\alpha=1$ (yielding $C=1$ ). After some further mathematical arrangements, we summarize the final results in the following:

$u_{1}(x, t)=\frac{2}{3} \frac{x}{t}+\frac{C}{m}$,

$h_{1}(x, t)=\frac{1}{9}\left(Q_{\alpha}^{2} g_{r}^{-\alpha}\right)^{\frac{1}{4-\alpha}}\left(3 C-m \frac{x}{t}\right)^{2}$,

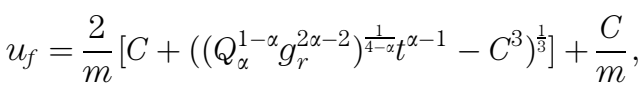

where $m$ is given in Eq. (5) and $C$ is given in Eq. (16). Equations (17) to (19) consist of the similarity solution for inviscid gravity currents without surface tension, while they are valid only when $\alpha=0$ or $\alpha=1$.

\section{Validation of the present solution}

There are two experimental studies with which the present results can be compared: one about dam-break currents conducted by Stansby, Chegini and Barnes [14], and one about lockexchange currents by Groebelbauer, Fannelop and Britter [15]. Both experiments were implemented under the constant flux condition $(\alpha=1)$, with which the similarity solution can be converted from Eqs. (14) and (16)-(19), yielding

$C=1$,

$u_{1}(x, t)=\frac{2}{3} \frac{x}{t}+\left(Q_{1} g_{r}\right)^{\frac{1}{3}}$,

$h_{1}(x, t)=\frac{1}{g_{r}}\left[\left(Q_{1} g_{r}\right)^{\frac{1}{3}}-\frac{1}{3} \frac{x}{t}\right]^{2}$,

$x_{f}=3\left(Q_{1} g_{r}\right)^{\frac{1}{3}} t$,

$u_{f}=3\left(Q_{1} g_{r}\right)^{\frac{1}{3}}$.

In the following two subsections, we will show that the present similarity solutions are in a good agreement with these experimental results.

\subsection{The dam-break current}

Stansby et al. [14] conducted a series of experiments to investigate the motion of dam-break currents and applied the shallow water equations (2) and (3) to simulate the current motion. They ended up with theoretical results fitting nicely with their experimental data, see, for example, their Fig. 8a, and their theoretical solutions turn out to be identical to the present similarity solution for $\alpha=1$ when an appropriate coordinate transformation is applied. To 
show this, we start from Eq. (22) and consider the solution at $x=0$, yielding $h_{1}(0, t)=Q_{1}^{\frac{2}{3}} g_{r}^{-\frac{1}{3}}=h_{0}$, so that

$Q_{1}=\sqrt{g_{r} h_{0}^{3}}$,

where $h_{0}$ is the height at the source $x=0$. By substituting $Q_{1}$ into Eqs. (21)-(24), we obtain the solutions in terms of $h_{0}$ as follows:

$u_{1}(x, t)=\frac{2}{3} \frac{x}{t}+\sqrt{g_{r} h_{0}}$,

$h_{1}(x, t)=\frac{1}{9 g_{r}}\left[\frac{x}{t}-3 \sqrt{g_{r} h_{0}}\right]^{2}$,

$x_{f}=3 \sqrt{g_{r} h_{0}} t$,

$u_{f}=3 \sqrt{g_{r} h_{0}}$.

From Eq. (26), the current speed at $x=0$ is found to be

$u_{1}(0, t)=\sqrt{g_{r} h_{0}} \equiv c$.

In the dam-break case, see Fig 2, Eq. (30) accounts for the backward-wave speed $c$ [4], [9], [14]. When the coordinate is fixed on this backward-wave, or equivalently, the coordinate transformation $\xi=x-c t$ is applied to Eqs. (28) and (29), we obtain

$\xi_{f}=2 \sqrt{g_{r} h_{0}} t$,

$u_{f}\left(\xi_{f}, t\right)=2 \sqrt{g_{r} h_{0}}$.

The above solutions (31) and (32) are the same as Eqs. (15) and (16) of Stansby et al. [14].

\subsection{Lock-exchange gravity currents}

Groebelbauer et al. [15] conducted a series of experiments for lock-exchange flows of different densities in a closed channel of square cross-section. They used different gases, such as air, argon, carbon dioxide, Freon 22 and helium, to produce the gravity currents of density ratios $\gamma$ varying from 0.9 (carbon dioxide in argon) to 0.05 (Freon 22 in helium), where $\gamma=\rho_{2} / \rho_{1}$. They also considered the influence of the depth ratio between the current and the channel, represented by the parameter $\Phi=h_{1} / H$ varying from $1 / 2$ to $1 / 6$. Since the gases were supplied by very large tanks, their cases shall be equivalent to the constant flux case $\alpha=1$. To make the

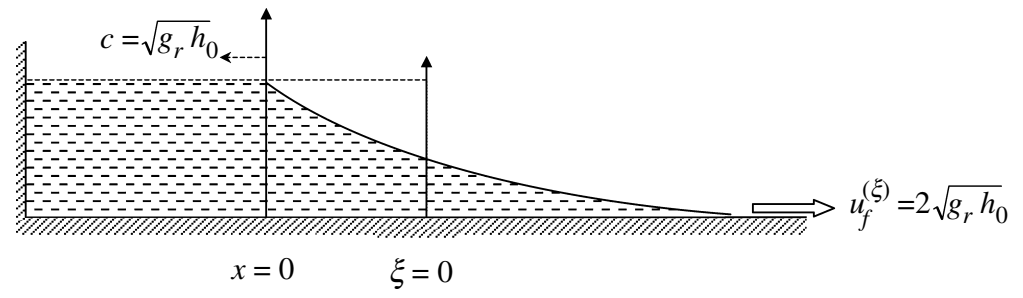

Fig. 2. A schematic description for the analogy between the gravity current and the forward-wave caused by dam-break. The dam was originally standing at the position $\xi=0$. As the dam breaks, it creates a forward-wave with a speed $u_{f}=2 \sqrt{g_{r} h_{0}}$ and a backward-wave with a speed $c=\sqrt{g_{r} h_{0}}$. As the coordinate is attached to the backward-wave, the analogy between forward-wave and gravity current persists 


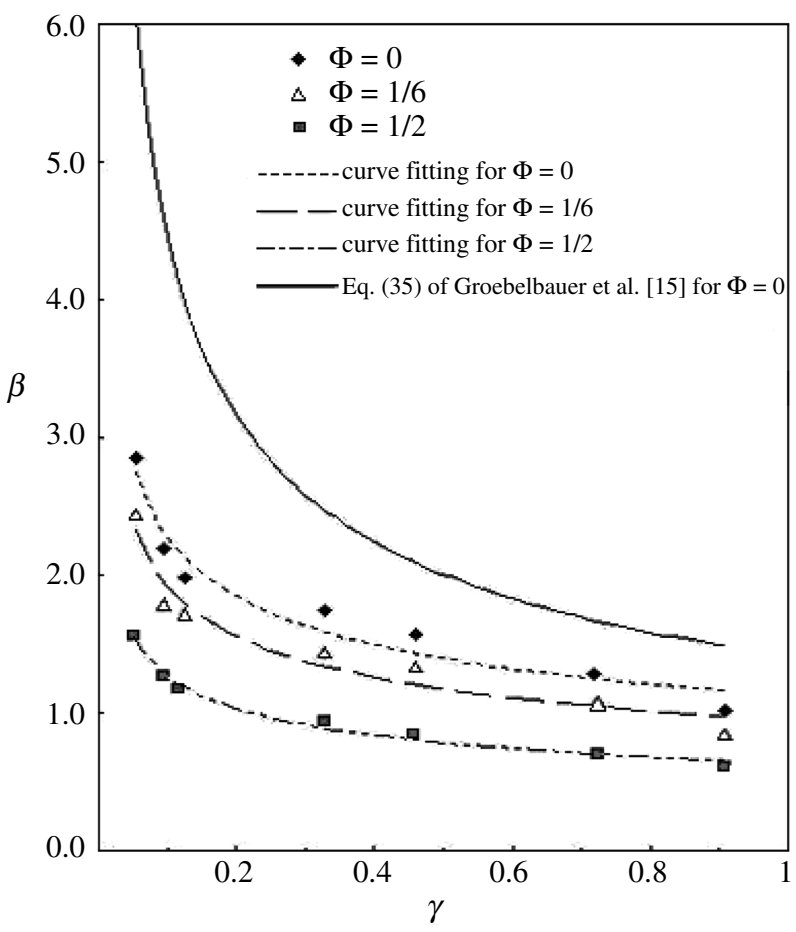

Fig. 3. The rearranged experimental data from Groebelbauer et al. [15] and the curve calculated from Eq. (35) of [15]. As $\gamma \rightarrow 0$, all cases show that $\beta \rightarrow \infty$ as obtained by the present approach

comparison easier, we convert their experimental data of their figs. 7, 8 and 10, accounting for the results of three different $\Phi$, into the values accounted for by the present parameters related to the parameters they used by the relations

$\gamma=\frac{1-\left(\rho^{*}\right)^{2}}{1+\left(\rho^{*}\right)^{2}}, \quad \beta \equiv \frac{u_{f}}{\sqrt{g_{r} h_{f}}}=\frac{u_{f}}{\sqrt{(1-\gamma) g h_{f}}}=\frac{F r}{\sqrt{1-\gamma}}$,

where, according to [15],

$\rho^{*}=\left(\frac{\rho_{2}-\rho_{1}}{\rho_{2}+\rho_{1}}\right)^{\frac{1}{2}}, \quad F r \equiv \frac{u_{f}}{\sqrt{g h_{f}}}$.

The converted data are shown in Fig 3, from which one can see that for all of the three different $\Phi$ considered the value of $\beta$ invariably increases exponentially with decreasing $\gamma$. Extrapolation of these data leads to a result that as $\gamma \rightarrow 0$ the value of $\beta$ approaches infinity, as predicted by the present solutions (20)-(24). Groebelbauer et al. [15], based on their experimental data, proposed an empirical relation for $\beta$ as a function of $\gamma$ and $\Phi$ such that

$\beta=\sqrt{\frac{1}{\gamma} \frac{(2-\Phi)(1-\Phi)}{1+\Phi}}$,

which also leads to $\beta \rightarrow \infty$ as $\gamma \rightarrow 0$. Note that Eq. (35) is in fact a modification of the formula proposed by Benjamin [1].

\subsection{The limit of the present solution}

The present solution (20)-(24) for $\alpha=1$ results in an infinite $\beta$ because the height of the current head vanishes (i.e., $h_{f}=0$ ), see Fig. 2 as well as Eq. (33). This kind of elongated 
wedge-like head has been observed by experiments, see for example, the spreading of oil over the sea surface (Simpson [2], pp. 106-111) or the lower-density current of the lockexchange flows (Groebelbauer et al. [15], p. 672). Theoretical results of the dam-break flow also give this wedge-like current (see fig. 8a of Stansby et al. [14]). The infinite $\beta$ leads to an important conclusion that the present similarity solution accounted for by Eqs. (14) and (16)-(19) is valid for the gravity current having a $\gamma \approx 0$, or for non-Boussinesq gravity currents. Klemp et al. [9] indicated that, when the ambient resistance imposed on the current front is taken into account by considering the front condition Eq. (1), the height of the current head becomes nonzero (see fig. $1 \mathrm{~b}$ of Klemp et al. [9]) or $\beta$ becomes finite. Accordingly, it is inferred that the elongated wedge-like current head is a result of the negligible resistance force from the ambient fluid. Due to the neglect of the ambient resistance, the present solution can be valid for the gravity currents moving in an ambient fluid of arbitrary height, as shown by the experimental data of [15] in Fig. 3 that, for all the depth ratios $\Phi$ considered, $\beta \rightarrow \infty$ as $\gamma \rightarrow 0$. In brief, the present similarity solution is valid for non-Boussinesq gravity currents moving in an ambient fluid of arbitrary height.

\section{Conclusions}

We have proposed a similarity transformation in Eqs. (4) and (5) to solve the shallow water equations (2) and (3) without needing to consider the front condition Eq. (1). We ended up with the similarity solutions valid for the inviscid non-Boussinesq gravity currents of both constant-volume $(\alpha=0)$ and constant-flux cases $(\alpha=1)$, and the results of the latter case were shown to be in a good agreement with the experimental results of the dam-break current [14] and the lock-exchange current of a much higher density than the ambient fluid [15]. We have also shown that the present similarity solutions can be reduced to those derived by Grundy and Rottman [7], and Gratton and Vigo [10] when appropriate conditions are imposed, although these two studies applied a different similarity transformation and had to use the front condition Eq. (1) to develop their solutions.

The major and possibly the most significant advantage of the present approach could be that, due to the absence of the front condition Eq. (1), we do not need to determine the parameter $\beta$ to develop our similarity solutions, and in fact $\beta$ becomes part of the present solution. The major disadvantage of the present approach is that the solution is only valid for non-Boussinesq gravity currents where the ambient resistance is negligible. This disadvantage is inevitable because, firstly, the shallow water equations (2) and (3) cannot govern the flow near the current head where most of the ambient resistance occurs, and secondly, the front condition Eq. (1) is not used so that the ambient resistance can not be taken into account by adjusting the value of $\beta$, which is usually determined according to varying physical situations. To investigate Boussinesq gravity currents where the ambient resistance is not negligible, the consideration of Eq. (1) may be a necessity.

\section{Acknowledgements}

This research has been supported by the National Science Council of Taiwan through grant NSC 91-2201-E-002-089. 


\section{References}

[1] Benjamin, T. B.: Gravity currents and related phenomena. J. Fluid Mech. 31, 209 (1968).

[2] Simpson, J. E.: Gravity currents in the environment and in the laboratory, 2nd ed. New York: Cambridge University Press 1997.

[3] Fay, J. A.: The spread of oil slicks on a calm sea. In: Oil on the sea (Hoult, D. P., ed.), pp. 53-63. New York: Plenum Press 1969.

[4] Fannelop, T. K., Waldman, G. D.: Dynamics of oil slicks. AIAA J. 10, 506 (1971).

[5] Hoult, D. P.: Oil spreading on the sea. Ann. Rev. Fluid Mech. 4, 341 (1972).

[6] Britter, R. E.: The spread of a negatively buoyant plume in a calm environment. Atmos. Environ. 13, 1241 (1979).

[7] Grundy, R. E., Rottman, J. W.: Self-similar solutions of the shallow-water equations representing gravity currents with variable inflow. J. Fluid Mech. 169, 337 (1986).

[8] Bonnecaze, R. T., Huppert, H. E., Lister, J. R.: Particle-driven gravity currents. J. Fluid Mech. 250, 339 (1993).

[9] Klemp, J. B., Rotunno, R., Skamarock, W. C.: On the dynamics of gravity currents in a channel. J. Fluid Mech. 269, 169 (1994).

[10] Gratton, J., Vigo, C.: Self-similar gravity currents with variable inflow revisited: plane currents. J. Fluid Mech. 258, 77 (1994).

[11] von Karman, T.: The engineer grapples with nonlinear problems. Bull. Am. Math. Soc. 46, 615 (1940).

[12] Simpson, J. E., Britter, R. E.: The dynamics of the head of a gravity current advancing over a horizontal surface. J. Fluid Mech. 94, 477 (1979).

[13] Maxworthy, T.: Gravity currents with variable inflow. J Fluid Mech. 128, 247 (1983).

[14] Stansby, P. K., Chegini, A., Barnes, T. C. D.: The initial stages of dam-break flow. J. Fluid Mech. 374, 407 (1998).

[15] Groebelbauer, H. P., Fannelop, T. K., Britter, R. E.: The propagation of intrusion fronts of high density ratios. J. Fluid Mech. 250, 669 (1993).

Authors' address: A.-C. Ruo and F. Chen, Institute of Applied Mechanics, National Taiwan University Taipei, Taiwan 106, Republic of China (E-mail: falin@iam.ntu.edu.tw) 